\title{
A NEW APPROACH TO REAL TIME TRAFFIC SIGN RECOGNITION BASED ON COLOUR INFORMATION
}

\author{
Lluís Pacheco, Joan Batlle, Xevi Cufi \\ UNIVERSITAT DE GIRONA \\ Escola Politècnica Superior \\ Dep. of Industrial Engineering
}

Avda. Lluís Santaló sn. 17003. Girona. Spain

Tel: 34.72 .418384$, Fax: 34.72 .41 .83 .99

\section{INTRODUCTION}

The detection and recognition of traffic situations has been a problem studied by an important number of researchers in the world. The classes of objects that are relevant for road vehicles are: recognition of road and its lanes, recognition of obstacles, detection of passing vehicles and following the own vehicle and, of course, the detection and interpretation of traffic signs.

[1] shows that the $3 \mathrm{D}$ description of the scene is enriched by detecting relevant features such as street signs (used to realize automatic warning systems for driving assistence, that is the main goal of our work), trees and light lamps usually placed at the right side of the road. Street signs have to espected shapes (octogonal, ciurcular, triangular and rectangular) and are usually located at standard height near the border of the road. The localization of landmarks starts with the detection of street signs, wich is performed by looking for their contours in the appropiate region of the image.

Then, for automatic co-pilot systems, the ability of traffic signs recognition is very important. Up to now, only a few systems are designed for traffic sign detection and interpretation.

[2] explains that edges and corners have been the most frequently used features for the extraction of objects in a car environment, but the texture and colour have been studied too. Using this features in addition with edges and corners, can provide redundancy, thus, increase the robustness of the system. In this work, one of the system part is the traffic signs detection module, that requires also two sub- modules: the detection sub-module and the interpretation sub-module. This group works mainly in the first sub-module, that detects the signs and track them until the image has reached a sufficient size, them takes a snapshoot of the sign that is the input for the interpretation sub-module.

[3] describes a hierarchical vision system for the detection and classification of traffic signs. The edges and color information of an image is used for a detection of traffic sign candidates. Then, the regions of interest (ROI), each of them containing one hypothetical traffic sign, are investigated using pixel classification methods. The authors shows some detectibility problems of traffic signs, especially when the signs are far away from the camera. In this case some distorsions of the interesting edge sequences ocur i.e. outlines of the signs are not complete and form part of large sequences of edges belonging to background objects. Robustness of the system can be enhanced by a combination of contour processing with the treatement of colour information of the candidates of traffic signs.

The approach described in [4] to TSR (traffic sign recognition) is based on a fast and stable color segmentation CSC (colour structure code) using also a new hierarchical region growing method. The system gather all detected objects of relevant colours for traffic signs and analyze their shapes. The system allows certain theresholds in the definitions of our colours of interest for the traffic signs and the results is a very robust TSR under all lighting conditions. The authors explains that at the moment the system don't identify the pictograms in the detected traffic signs.

[5] describes a system in wich a colour images are acquired by a camera mounted in a car. The images are also segmented based on colour. These combinations generate hypotheses for traffic signs and then are verified using a pictogram classifier. Colour information in contrast to shape information are invariant against scale and view, and possess highly discriminative power especially in the domain of traffic sign detection. 
All this work shows that the interpretation of conventional traffic signs is very complex and time consuming.

The system set up by our work group is am "automatic warning system for driving assistance" which only considers the aspect of the detection and interpretation of traffic signs. Our system does not claim to be able to interpret the standard traffic signs on the roadside, the proposal is to incorporate into the existing signs another type of traffic sign whose information will be more easily interpreted by a processor. The type of information to be added to these signs is profuse and therefore the most important object is the robustness of the system. The basic proposal of this new philosophy is that the co-pilot system for automatic warning and driving assistance can interpret with greater ease the information contained in the new sign, whilst the human driver only has to interpret the "classic" sign.

One of the codings that has been tested with good results and which seems to us easy to implement, is that which has a rectangular shape and 4 vertical bars of different colours, as shown in Fig. 1. The size of these signs is equivalent to the size of the conventional signs (approximately $0.4 \mathrm{~m} 2$ ). As can be appreciated in the references mentioned, the colour information from the sign can be easily interpreted by the proposed specific processor and the interpretation is much easier and quicker than the information shown by the pictographs of the classic signs.

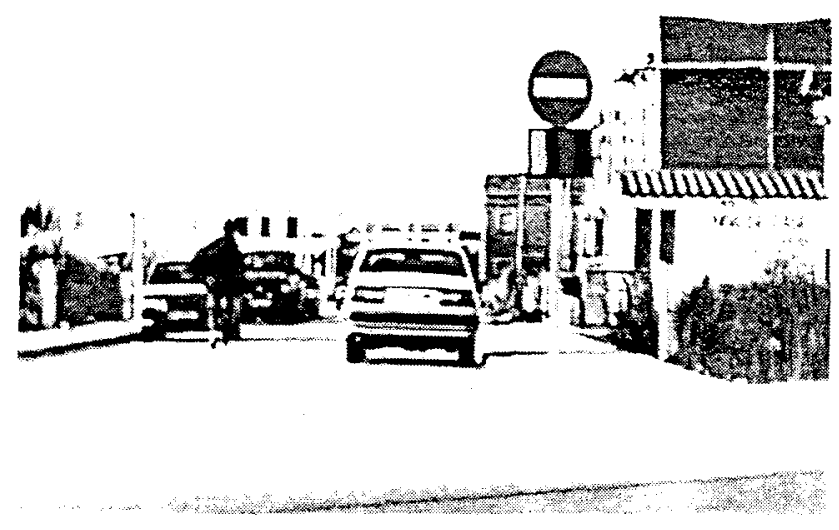

Fig. 1 Sign of rectangular shape with 4 verticals color bars

These new signs with coloured bars have little sense for the human driver as the information shown by the bars on a rectangular shaped background does not make learning and remembering easy, as there is no pictographic information.

We would like to point out that the economic cost for the authorities to set up this type of profuse signalling is not significant when compared to other investments made in similar situations.

\section{SYSTEM and PROBLEM DESCRIPTION}

The working of the system is based on the capture of a line in any part of the image, from the video sign coming from the camera, which has the colour characteristics which are integrated in the new traffic signs. When the system scans the image, the sign is in some part of the image, it will find the colour code values of each one of the 4 vertical bars contained in the sign. Another valued characteristic is that each one of these colour code values (with prefixed margins of error) is found in the line of the video sign, during a set quantity of pixels, with values similar to each one of the zones occupied by each colour bar. To explain this we give an example:

One can see in Fig. 2 that starting from any pixel of the image (ij) we can find the coding value of the colour blue ( 90 $+/-5$ ) for 81 pixels of the sign line of the video (which is being scrutinized at this moment). Afterwards, during 83 pixels the coding value of the colour red $(245+/-5)$ is found, immediately afterwards for 79 pixels the coding value of the colour yellow $(180+/-5)$ is found and then for 77 pixels the coding value of the colour green $(150+/-5)$ is found. In this case a sign line will have been found made up of 4 vertical bars: blue, red, yellow and green in that order and which correspond to a standard sign in the Highway Code.

In this example, apart from the colour signs corresponding to the 4 bars of the sign, in the line scrutinized there could appear more information about the background colour. This information may or may not contain coding values of colours equal to those found in the sign bars, thus there may be objects present in the image with the same colour as 
one of the bars. What is practically impossible to occur is that in the background (in the rest of the colour information of the image) the coding colour values permitted in the sign bars is repeated with the same pattern of width regularity as is found in the signs.

The width values for the detection of the colour signs (the values $81,83,79$ and 77 of the example) depend on the distance from which one can consider that the detection of the sign is good. The system only takes into account the width values sufficiently large for the validation of the sign detection (it is assumed that the sign is sufficiently near to the vehicle) and from a certain prefixed minimum threshold in the testing and calibration procedure of the system.

A system which is viable for the improvement of driving safety must be simple, economic and work in real time. These are the characteristics of the scheme proposed.

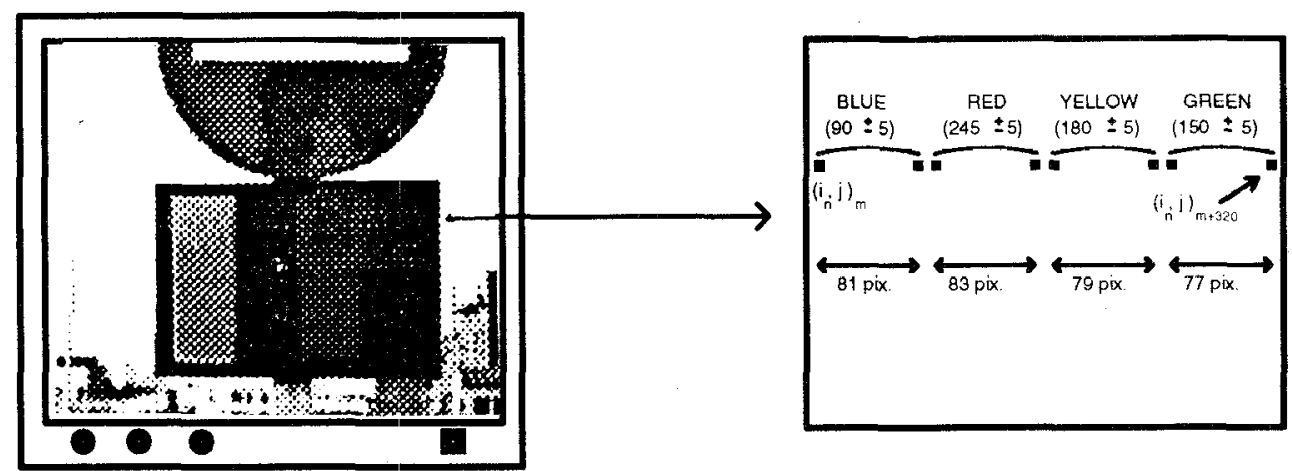

Fig. 2 Values and wide of a vertical bar sign

A system made up of two basic working modules is used. The first is for the conversion of RGB/HSI coordinates which allows the segmentation of the colour information to be carried out simply and in real time. This data is the input to the second module of the system, a specific processor used for the interpretation of traffic signs as is shown in the following paragraph.

The RGB/HSI conversion can be made using a series of colour coordinate conversion models which have been defined by different authors. The conversion model used in our system is that proposed in [6] where we have chosen for the alternative signs those colours which have the most stable behaviour when faced with changes in the luminosity [7] This model is the one which, during trials, has shown to have the best behaviour of all those which were tried in environmental conditions which could be considered normal (daytime driving, the weather not excessively sunny, the existing signs in good condition, without reflections in the signs, background image without excessive colour information...).

The colour segmentation procedure uses 2 of the 3 output components which the RGB/HSI conversion stage supplies as there could happen that, under certain environmental work conditions of the system, the use of only one output component may be insufficient for the carrying out of the appropriate segmentation. The use of the resultant $\mathrm{H}$ and $\mathrm{S}$ colour components in the 2nd sign detection and interpretation module makes the system more robust, as can be seen in Fig. 3, as the colour coding by the sign detection is carried out independently in the two components. As can also be seen in this figure the contrast obtained in the luminescence component $\mathrm{L}$ is inferior.

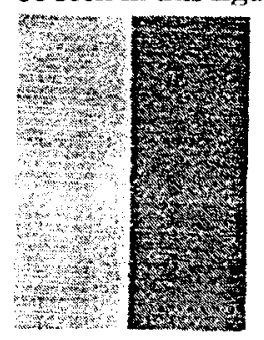

Hue
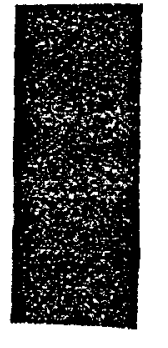

\section{Saturation}

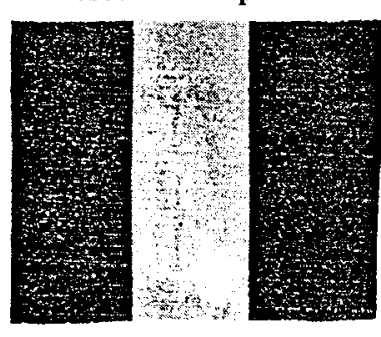

Fig. 3 Hue, Saturation, and Luminance of a traflic sign intage.

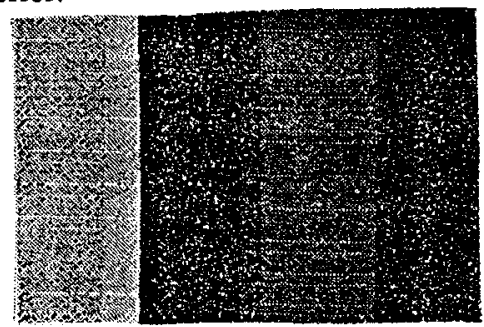

Luminance 


\section{HARDWARE ENVIRONMENT}

Our hardware system is based on two modules: conversion RGB/HSI module and the Specific Processor for Traffic Sign Recognition. These two modules can work alone or configured, controlled and supervised by a host computer.

\subsection{RGB/HSI MODULES}

The entrance of the video signal is provided by a camera located on the top of the car., where there is the best field of vision. The fact that the lighting of the driving conditions changes rapidly (shadows, tunnels, brightness, etc.) is an important argument to consider when choosing the camera. Another interesting point for the choice of the camera is the RGB/HSI conversion done by this first module. So, we need a CCD camera with the three basic analog components and the sync. signal, including autoiris function or automatic control gain set in function of the brightness.

In Fig. 4, we show the bloc diagram of this module. The idea is that after the analog to digital conversion, we do an adress bus making the union of the three digital signals $R, G$ and $B$. With this digital bus, we have three static memories fitted with the HSI information so they work like three LUTs, one for each component.

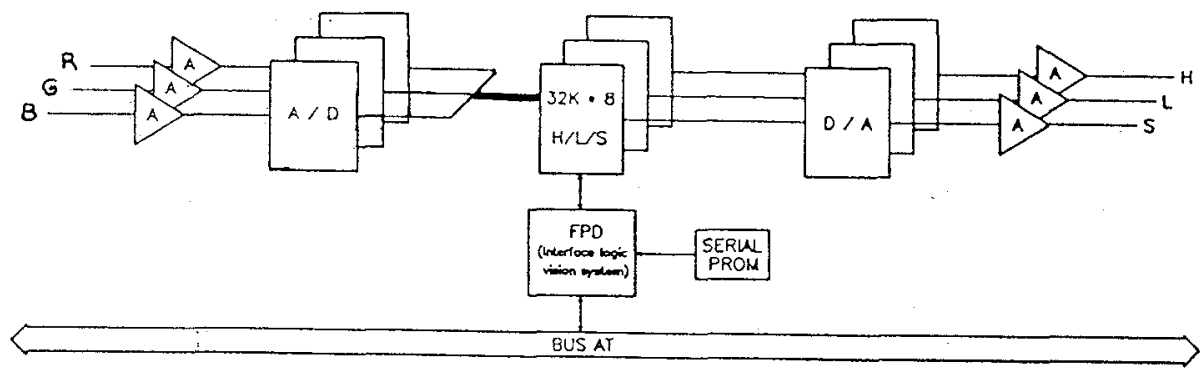

Fig. 4 RGB/HSI module.

If we had decided to use the mathematical model of conversion, we could work with this modulealone thus writing the information to the three memories (EEPROM or FLASH). If we work with the host computer, we can use their bus to write the memories and other tasks of supervision. In this way we can easily test the different mathematical models.

The resolution for our PCB is 5 bits for each colour (15 bits of adress bus), but with the actual technology, it is very easy to change the system to 8 bits of resolution for each colour, (24 bits of adress bus). For all our applications we have enough robustness with 5 bits.

The most important sub-modules are: input A/D converters, output D/A converters, static memories and sync. generator. The control and interface with the host is done using a Field Programmable Gate Array.

\subsection{SPECIFIC PROCESSOR FOR TRAFFIC SIGN RECOGNITION}

The two inputs for this module are Hue and Saturation components, wich are the most robust signals under luminosity changes. These signals are converted to digital and then processed by a state machine.

We have developed some experimental colour signal codings. One of the multiple possibilities implemented is a coding placed in a border of a sign with a specific colour and four different colours (red, green, blue, yellow) used to identify the different signals. With four different colours we can codify in a binary form, 24 signals.

Fig. 5 shows the system bloc diagram. First, we converted the analog Hue and Saturation to digital. After this, the Machine sees if the digital value (hue and saturation) has a value between the maximum and minimum threshold acceptable for the colour border (beginning of the code signal). If this is true, the system begins to read the code and enable an internal flag of "signal reading". It reads the border colour, but after it begins to read the four code colours, it should have an internal flag for each of the colours for the order of arrival and codification of the signal. Four full adders are increased if the digital value is between the selected thresholds, the system remains in this state until it 
reads the end border colour or the end of the video signal line. If the border colour is read, the flag "sign reading" is disabled. When the signal has been read the system looks for its validity.

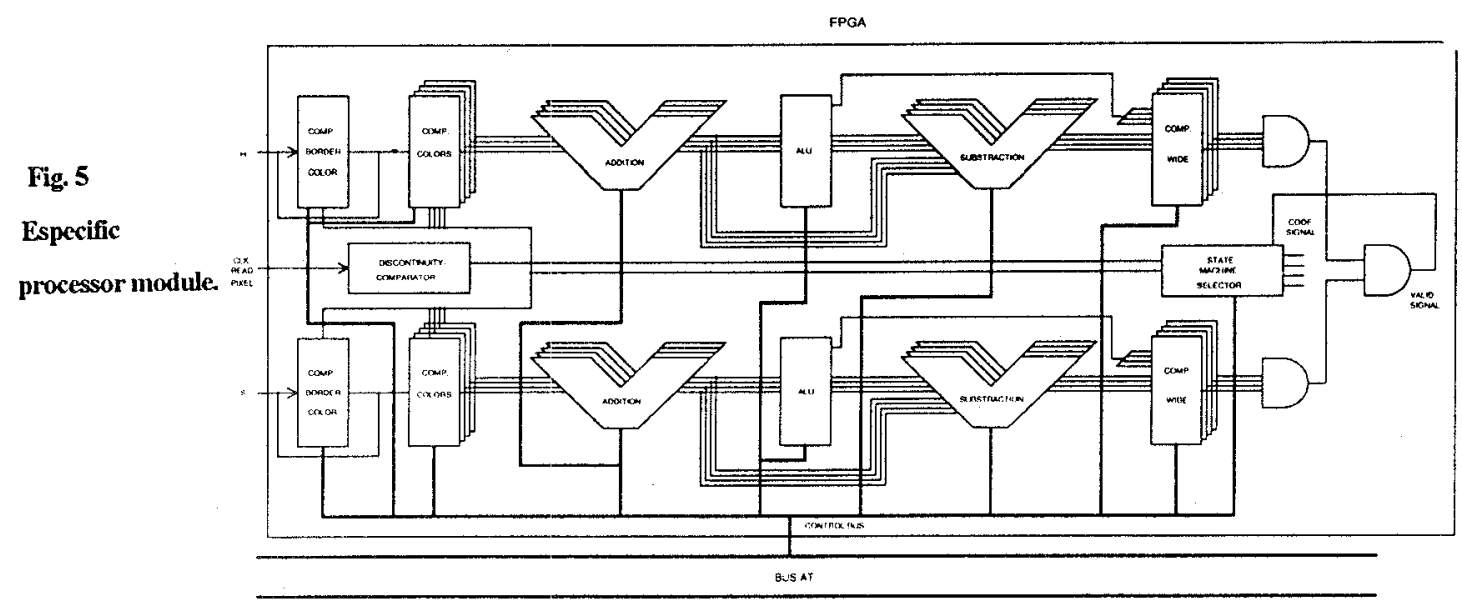

The block called ALU adds the four colours and divide them by four and it calculates the average value and also it calculates the tolerance of the sign that it will be a selectable percentage. The average value is compared with the four values of the full adders. If the difference is less than the tolerance, the code signal is valid. Then the system produces an output of the codification of the signal and sets up the validity flag.

The discontinuity control block only works when the signal reading flag is set, and sets up a counter to be increased by one each time that the pixel reading is not identified as colour of the sign. So when the internal flag for each colour are modified, the counter will be read and the value should be less than a selectable tolerance value. If the counter value is greater than the tolerance, the sign will be invalid. After the reading of the counter it must be reset as another colour starts.

The reset of the system will be done if it produces the conditions: discontinuity, an invalid sign or the end of the video signal line. The activation of the discontinuity control blck is done by the flag "signal reading", and the value of the hue and saturation pixel colour is different to all the colours looked for.

We have used a Hardware Description Language to program the FPGA which implements the State Machine. If the recognition is $\mathrm{OK}$, an output with the code of the signal and a validity bit are provided.

The host can read this information about the signal and it can give the information to the driver. It can also configure the selectable parameters.

The use of the HDL and FPGA technology supply an easy way for debbuging different systems and codes. The fact of having an interface with the host reinforce the adaptability and intelligence of the system.

\section{CONCLUSION AND FUTURE EXTENSIONS}

We think that the system is appropriate as an additional security measure given that absolute security can never exist.

The calibration and working tests of the system were made in a closed circuit to avoid interrupting other traffic.

The system that we propose has a reliability of almost $100 \%$ in the weather conditions that we consider to be normal. The system has reacted correctly, including in light rain conditions. The system is also reliable in night driving as the lights of the vehicles illuminate the traffic signs and sign boards in such a way that our system is capable of capturing the colour information in these signs.

The authors consider that in a not too distant future, road safety will be increased if a type of signposting which can be computer interpreted is used. The system suggested in this paper is a first approximation to this as the colour information which is used is easily interpreted and processed by a computer. Thus the solution that we propose is simple, cheap and robust. 
The improvement in the colour coding system used must be a motive for future studies, as also the best siting of traffic signs with the object that the system be more reliable in adverse driving and road conditions.

An example of this would be the horizontal siting of an alternative type of traffic signs which would have these principal advantages: fixed vehicle-sign distance and a fixed background image with little colour information. The most important disadvantages which this model would have would be: the maintenance of the signalling and the siting of the camera on the vehicle.

The authors also believe that to achieve better degrees of safety it is necessary that in certain zones (principally urban) the system must be integrated with the environment.

These reflections encourage us to continue working in the field of driving safety and to develop future ideas.

\section{REFERENCES}

[1] "Visual Routines for Outdoor Navigation". Campani, Cappello, Piccioli, Reggi, Straforini, Torre. Proc. on I.V.'93.

[2] "Vision for Intelligent Road Vehicles". Graefe. Proc. on I.V.'93.

[3] "Interpretation of Traffic Scenes using a Hierarchical Data Structure". Mertsching, Austermeier, Büker, Hartmann. Proc. on I.V.'93.

[4] "Traffic Sign Recognition based on Color Image Evaluation". Priese, Rehrmann, Schian, Lakmann, Bilderkennen. Proc. on I.V.'93.

[5] “Hybrid Approach for Traffic Sign Recognition”. Jansen, Ritter, Stein, Ott. Proc. on I.V.'93. configured, controlled and supervised by a host computer.

[6] "Segmentation of Color Aerial Photographs using HSV Color Models". Yagi, Abe, Nakatani. MVA'92 Workshop on Machine Vision Applications. Tokyo.

[7] "Aportació a la Detecció de Moviment Independentment del Moviment de la Càmera, basat en el Processat d'Imatges en Color". J. Batlle. Dep. ESAII. Universitat Politècnica de Catalunya. Barcelona 1993. 